\title{
Some Unique Considerations in Treatment of Multiple Sclerosis
}

\author{
Faizan Mazhar" and Nafis Haider
}

Basic Medical Science Unit, Prince Sultan Military College of Health Sciences, Dhahran, Saudi Arabia; mfznbaig@gmail.com

\begin{abstract}
Multiple Sclerosis (MS) is one of several diseases described as demyelinating because it causes damage to the myelin sheath. The presence of additional medical conditions like pregnancy, osteoporosis and infections are common with multiple sclerosis that adversely affects the health outcomes. The treatment of MS becomes more complex when compounded by these existing additional medical conditions. This review highlight important pharmacotherapeutic considerations in treatment of MS in these special patient population.
\end{abstract}

Keywords: Immunization, Multiple Sclerosis, Osteoporosis, Pregnancy

\section{Introduction}

Multiple Sclerosis (MS) is a multifactorial CNS disease characterized by a relapsing-remitting course and continual accumulation of neurologic deficits. This disease affects more than 2 million people worldwide, primarily women, with onset occurring in young adults (age 20-40 years). The etiology of the disease is unknown but considered to be a combination of environmental and genetic factors. Risk factors for MS include EpsteinBarr virus, low concentrations of vitamin D, obesity, mononucleosis, increased sodium intake, and cigarette smoking ${ }^{1}$.

Multiple sclerosis is potentially triggered by an autoimmune process that affects the CNS. The disease causes inflammation of both white and grey matter. The typical features of MS are widespread areas of demyelination noted as plaques or lesions $s^{2}$. Remyelination occurs over several months after lesion formation and is extremely variable between patients and in different plaques within the same patient. The process becomes slower over time as the disease progresses. Remyelination is highly inefficient and does not lead to full axonal integrity, thus leading to disease progression ${ }^{1}$. The specific management questions and concerns surround in patients with special conditions. This is complicated by the fact that MS has entered a treatment era. Unfortunately, there are no recognized and implemented guidelines. The present review highlight important pharmacotherapeutics considerations in unique situations of multiple sclerosis.

\section{Pregnancy}

Multiple sclerosis predominately affects women and often has a clinical onset during childbearing years. Therefore, for women with child bearing potential diagnosed with MS, reproduction is an important consideration. The effect of pregnancy on relapse risk of MS is the most studied aspect. Studies have shown that the relapse rate over the whole pre-pregnancy to post-pregnancy period appears to be identical to that of the nonpregnant state. In the recent past, pregnancy has been reported to have both negative effects or benefit to maternal MS progression. Women with progressive forms of MS are less likely to have children, creating a limitation in this area of study. In these women change in fertility rates have not been demonstrated by reliable studies.

* Author for correspondence 
Individuals who wish to have children should be encouraged to consider strategies to increase the probability of conceiving during the period they are relatively healthy and not taking any Disease Modifying Treatments (DMTs) ${ }^{3}$. Another concern for women with MS is the risk of transmission of disease to their children. Compared to general population, offspring of women with MS have a 20-50 times greater risk of developing MS, although the absolute risk is relatively low ${ }^{4,5}$.

Glatiramer acetate (FDA pregnancy category B) does not appear to be associated with teratogenic risk or a higher risk of miscarriage ${ }^{6}$. Fingolimod is classified as FDA category $C$ (i.e., animal studies warrant patients be counselled on foetal risk and appropriate contraception use for at least 2 months after stopping the drug). Natalizumab is also FDA category $\mathrm{C}$ based on the lack of human data $^{6}$. Mitoxantrone and teriflunomide are associated with teratogenic effects and should only be prescribed to women who are using reliable contraception ${ }^{7,8}$. Based on the available literature, glatiramer acetate is the safest option during pregnancy if a DMT is necessary $y^{6}$. Typically, patients are counselled on stopping their DMTs immediately before and during pregnancy.

Experts generally recommend a 2-6-week discontinuation of therapy before attempting to conceive, with the exception of fingolimod and teriflunomide. Fingolimod may take up to 2 months to be cleared, and teriflunomide can take up to 2 years. If a man or woman is considering starting a family, cholestyramine $8 \mathrm{~g}$ every 8 hours for 11 days should be admin-istered after discontinuation of teriflunomide. Contraceptives must be continued as long as the teriflunomide serum concentration is greater than $0.02 \mathrm{mg} / \mathrm{L}^{9}$.

A serious MS relapse generally treated by either intravenous corticosteroids or intravenous immunoglobulin (IVIG). Steroids increase the risk of preeclampsia, premature rupture of membrane, maternal hypertension and infections. Intravenous immunoglobulin has no known teratogenic effects and appears to have no effects on the immune system of the foetus. The optimal timing of restarting therapy or initiating breastfeeding is unclear. Research has shown interferon-beta, glatiramer acetate, and natalizumab to be moderately safe during breastfeeding. However, with the limited information available, women should not resume therapy until after breastfeeding. For lactating mothers, corticosteroids or IVIG are the options for relapse prevention or treatment. Monthly corticosteroids have been used to reduce the risk of postpartum relapses because corticosteroids are relatively safe to use but do have adverse effects in children ${ }^{10}$. It is recommended to suspend breastfeeding for 4 hours after infusion of a corticosteroid with a "pump and dump" approach. Maternal IVIG use does not seem to have detrimental effects on the infant ${ }^{4,11}$.

\section{Osteoporosis}

Osteoporosis is more common in patients with MS than the general population. Reasons for this increased risk include the use of corticosteroids, immobility, and the increased probability of falls because of gait difficulties. Multiple sclerosis has also been associated with low amounts of vitamin $\mathrm{D}$ and smoking, two additional risk factors for osteoporosis. Interferon-beta may have favourable effect on bone metabolism in patients with MS, probably because of the inhibitory effect of interferonbeta on osteoclasts ${ }^{12}$. The Fracture Risk Assessment Tool (FRAX) tool is routinely used in practice to establish the risk of osteoporosis-related fractures; however, it likely underestimates the risk in patients with MS because co-morbidity is not used in calculating fracture risk. Regardless, the reduction in bone mineral density may have already started at the time of initial clinical presentation. Therefore, patients should be proactive to ensure optimal bone health ${ }^{13}$.

\section{Dietary Considerations}

Dietary interventions can favourably influence MS; proposed interventions included diets high in polyunsaturated fats and low in animal fats, gluten-free, sucrose-free, pectin-restricted diets, and the Paleo diet. Vitamin B12 deficiency may lead to deformed myelin and is associated with immunomodulatory activities. Low concentrations of vitamin B12 have been reported in patients, but a relationship between vitamin B12 and MS has yet to be confirmed ${ }^{14}$. Supplements with antioxidant activity (vitamin A, C, E and Co-enzyme Q 10) may decrease free radical-induced cellular injury, which may con-tribute to myelin and axonal injury ${ }^{15}$.

Omega- 3 fatty acids may have clinical and biochemical effects in patients with MS. This supplement may 
have anti-inflammatory, antithrombotic, antioxidant, immuno-modulatory functions, as well as neuroprotective effects $^{16,17}$. Because increased oxidative damage has been associated with disease progression, a reduction of proinflammatory cytokines and oxidative stress could benefit patients. Taking 4 gram of fish oil daily has been shown to reduce inflammatory cytokine concentrations. However, the supplement has not been proved to reduce relapse rates, disability progression, or fatigue ${ }^{15}$.

Vitamin D has been linked to the risk of developing MS through evidence demonstrating that individuals with MS have lower levels of 25-hydroxyvitamin D relative to healthy controls ${ }^{18}$. Bioactive vitamin $\mathrm{D}$ may modulate the production of neurotrophins, growth factors, and neurotransmitters, as well as the induction of nerve growth factor syntheses. Several studies investigating the effect of vitamin D to modify the course of MS have had inconclusive results ${ }^{18}$. More evidence favours vitamin D supplementation as a preventive measure in developing MS. The optimal dosage and age to target using vitamin D supplementation remains unclear.

\section{Immunization}

Evidence has suggested that MS exacerbations occur because of infectious episodes, some of which may be prevented by vaccination. However, concerns about the safety of vaccinating patients with MS remain ${ }^{19}$. The immunization panel of Consortium of Multiple Sclerosis Centers in collaboration with American Academy of Neurology, published a summary of evidence and recommendations regarding immunizations and MS. Hepatitis B, inactivated influenza and tetanus vaccines are safe for patients with MS. In general, inactivated vaccines are considered safe; however, live attenuated vaccines are not recommended with the exception of live zoster vaccine. Live Varicella vaccine should be considered in a patient who has never had Varicella Zoster Virus (VZV) infection, lacks evidence of prior immunity, or is considering starting an MS therapy that suppresses cell-mediated immunity. Pneumococcal vaccine is safe for patients with MS. Immunization with the VZV live virus vaccine should be given 2 months before initiating fingolimod, regardless of whether a patient is receiving an immunomodulatory DMT.
If a patient is experiencing a serious relapse, vaccination should be deferred until 1-2 months after the onset of relapse. Patients on chronic corticosteroid treatment should consult with their neurologist before taking a live vaccination. If a close family member receives a live vaccine, a neurologist should be consulted regarding what precautions the patient with MS on immunosuppressant therapy should take ${ }^{20}$.

\section{Oral Hygiene}

Having MS can affect the ability of patients to maintain oral hygiene, preventive maintenance and seeking dental care. Patients with paraesthesia in arms and hands will have a decreased ability to carry out adequate oral hygiene. Many drugs used to treat MS have adverse effects such as gingival hyperplasia, xerostomia, and stomatitis; oral vigilance is therefore necessary in these patients. It is optimal that the dental treatment should be performed when the patients' disease is in remission state. Appointments should be scheduled more often, for shorter durations, and in the morning, all of which reduce stress in the patient with neurologic problems.

\section{Conclusion}

An integrated multidisciplinary health care team approach is essential in the management of MS in special patient population. Appropriate selection and evaluation of pharmacotherapy is crucial. Careful selection of treatment options, encouraging modification of risk factors and providing contraceptive and preconception counselling are all important contributions to care which can maximize the treatment outcomes and help patients set realistic expectations about their MS treatment.

\section{References}

1. Inglese $M$, Petracca $M$. Therapeutic strategies in multiple sclerosis: A focus on neuroprotection and repair and relevance to schizophrenia. Schizophrenia research. 2015; 161(1):94-101.

2. Coyle PK. Management of women wilth multiple sclerosis through pregnancy and after childbirth. Therapeutic Advances in Neurological Disorders. 2016; 9(3):198-210. 
3. Devonshire $\mathrm{V}$, et al. The immune system and hormones: review and relevance to pregnancy and contraception in women with MS. International MS journal/MS Forum. 2003.

4. Bove $\mathrm{R}$, et al. Management of multiple sclerosis during pregnancy and the reproductive years: a systematic review. Obstetrics \& Gynecology. 2014; 124(6):1157-68.

5. van der Kop ML, et al. Neonatal and delivery outcomes in women with multiple sclerosis. Annals of neurology. 2011; 70(1):41-50.

6. Salminen HJ, Leggett H, Boggild M. Glatiramer acetate exposure in pregnancy: preliminary safety and birth outcomes. Journal of neurology. 2010; 257(12):2020-3.

7. De Santis M, et al. The first case of mitoxantrone exposure in early pregnancy. Neurotoxicology. 2007; 28(3):696-7.

8. Warnke C, et al. Review of teriflunomide and its potential in the treatment of multiple sclerosis. Neuropsychiatr Dis Treat. 2009; 5:333-40.

9. Kappos L, et al. Oral fingolimod (FTY720) for relapsing multiple sclerosis. New England Journal of Medicine. 2006; 355(11):1124-40.

10. Confavreux C, et al. Rate of pregnancy-related relapse in multiple sclerosis. New England Journal of Medicine. 1998; 339(5):285-91.

11. Dwosh E, Guimond C, Sadovnick A. Reproductive counselling for MS: a rationale. International MS Journal/MS Forum. 2003.
12. Chaudhuri A. Why we should offer routine vitamin D supplementation in pregnancy and childhood to prevent multiple sclerosis. Medical hypotheses. 2005; 64(3):608-18.

13. Dobson R, Ramagopalan S, Giovannoni G. Bone health and multiple sclerosis. Multiple Sclerosis Journal. 2012; 18(11):1522-8.

14. Cassard S, et al. Multiple sclerosis patients differ from healthy controls on antioxidant and anti-inflammatory nutrients from self-reported diet history (P2. 209). Neurology. 2015; 84(14 Supplement):2-209.

15. Farinotti M, et al. Dietary interventions for multiple sclerosis. Cochrane Database Syst Rev. 2012; 12.

16. Swank R, Dugan BB. Effect of low saturated fat diet in early and late cases of multiple sclerosis. The Lancet. 1990; 336(8706):37-9.

17. Swank RL, Dugan BB. The multiple sclerosis diet book. Doubleday; 2011.

18. Duan S, et al. Vitamin D status and the risk of multiple sclerosis: a systematic review and meta-analysis. Neuroscience letters. 2014; 570:108-13.

19. Goldman MD, Naismith RT. Multiple sclerosis, immunomodulation, and immunizations Balancing the benefits. Neurology. 2015; 84(9):864-5.

20. Rutschmann OT, et al. Immunization and MS A summary of published evidence and recommendations. Neurology. 2002; 59(12):1837-43. 